\title{
Arbeitsschwerpunkt: Forschung
}

\author{
ALS FORSCHERIN IN EINER REHAKLINIK Studien durchzuführen ist das Ziel vieler \\ Physiotherapiestudenten. Corina Schuster hat es erreicht: Die Doktorandin arbeitet \\ zu $80 \%$ in der Forschung. Nicht an einer Universitätsklinik, sondern in einer \\ Schweizer Rehaklinik.
}

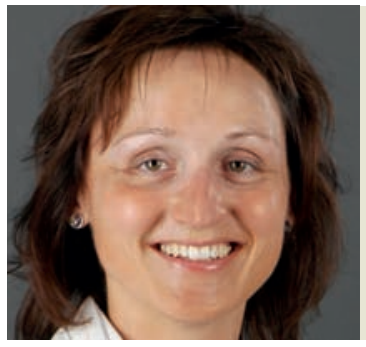

Corina Schuster ist seit 1997 Physiotherapeutin. Seit zehn Jahren lebt sie in der Schweiz und arbeitet in der Reha Rheinfelden, einer Klinik mit dem Schwerpunkt Neurologie und Orthopädie. Mittlerweile hat sie den Mastertitel erworben, ist stellvertretende Leiterin der Forschungsabteilung und promoviert an der Oxford Brookes Universität in Großbritannien.

Für ihre Doktorarbeit untersucht sie derzeit die Wirkung des mentalen Trainings auf Patienten nach Schlaganfall.

Frau Schuster, wie kamen Sie zur Forschung? Als ich anfing, in der Rehaklinik zu arbeiten, bestand die wissenschaftliche Abteilung bereits. Als meine Kollegen irgendwann Physiotherapeuten suchten, die im Rahmen einer Studie bei den Probanden motorische Assessments durchführen sollten, habe ich mich gemeldet. Das war mein Einstieg in die Forschung. Nachdem eine Stelle in der wissenschaftlichen Abteilung frei wurde, hatte ich die Möglichkeit, das wissenschaftliche Arbeiten zu vertiefen. Zunächst habe ich zu etwa $80 \%$ mit Patienten gearbeitet und zu $20 \%$ in der Forschung. Mittlerweile ist es umgekehrt.

\section{Wie ist die wissenschaftliche Abteilung in die Rehaklinik eingebunden?}

In dieser Abteilung arbeiten Ärzte und Physiotherapeuten und - je nach Projekt - zum Beispiel auch Ergo- und Musiktherapeuten. Der Bereich ist dem Chefarzt unterstellt, der auch Abteilungsleiter ist. Keiner meiner Kollegen ist in Vollzeit als Wissenschaftler tätig. Bei der Vorbereitung und Durchführung von wissenschaftlichen Untersuchungen arbeiten wir selbstständig, und bei der Datenauswertung unterstützt uns ein externer Statistiker.

\section{Welche Forschungsschwerpunkte haben Sie?}

Wir beschäftigen uns hauptsächlich mit Therapiemöglichkeiten bei Patienten nach Schlaganfall. Bisher sind es 13 Projekte. Ein Großteil sind Medikamenten-, Therapie- und Validierungsstudien. Die Designs sind sehr unter- schiedlich und in der Regel quantitativ angelegt: Fallstudien, Querschnittsuntersuchungen und randomisierte kontrollierte Studien.

\section{Wie finanziert die Abteilung die Studien?}

Wir haben drei verschiedene Finanzierungsquellen: die Klinik selbst, Stiftungen und Fonds, sowie Dienstleistungen für externe Institute.

Welche Vor- und Nachteile bringt die Forschung in der Klinik mit sich?

Ein wesentlicher Vorteil gegenüber Hochschulen ist die Nähe zu den Patienten. In Zusammenarbeit mit Patienten und Kollegen können wir sehr praxisnahe und patientenorientierte Fragestellungen entwickeln. Außerdem bieten uns die Infrastruktur und das Personal der Klinik einen sicheren Rahmen für die Durchführung von Patientenstudien. Darüber hinaus kommen die Forschungsergebnisse unseren Kollegen zugute, da wir unsere Erkenntnisse möglichst oft in internen Fortbildungen weitergeben. Ein Nachteil ist, dass es für uns im Vergleich zu den Hochschulen deutlich schwieriger ist, Drittmittel einzuwerben.

Momentan sind Sie in Oxford und arbeiten an Ihrer Doktorarbeit. Was untersuchen Sie?

Die Wirkung von Bewegungsvorstellung auf das motorische Lernen bei Patienten nach Schlaganfall. Zwei Patientengruppen erhielten in zwei Wochen sechs Therapieeinheiten, in denen sie den Bewegungsablauf „Boden- transfer und wieder aufstehen" erlernten. Danach sollten sie den Ablauf aktiv und mental trainieren, jede Gruppe nach einer anderen Methode. Weitere 14 Personen gehörten der Kontrollgruppe an, die eine Audioschulung zum Thema Schlaganfall bekam. Den Bewegungsablauf habe ich mit verschiedenen Assessments wie der Berg Balance Scale, der benötigten Zeit und anhand der Fehlerzahl beurteilt. Um herauszufinden, wie die Patienten das mentale Training erleben, habe ich sie außerdem interviewt.

Können Sie schon etwas zu den Ergebnissen sagen?

Nein, die Analyse läuft noch. Was ich sagen kann, ist, dass die Probanden die Technik der Bewegungsvorstellung schnell gelernt haben und es nach der Studie auch auf andere Bewegungsabläufe anwenden konnten.

Wie ist das Promotionsstudium in England organisiert?

Die Studie führe ich in unserer Rehaklinik durch. Sechs Wochen im Jahr bin ich in Oxford. Mein Aufenthalt wird von der schweizerischen Gottfried und Julia Bangerter-Rhyner Stiftung und der Klinik unterstützt. Während dieser Präsenzphase besuche ich verschiedene Module an der Uni und führe Gespräche mit meinen Supervisoren und Kollegen: Ergotherapeuten, Psychologen, Krankenschwestern und Sozialarbeitern.

Haben Sie zum Schluss noch Tipps für Physiotherapeuten, die gerne forschen möchten?

Unerlässlich ist es, sich ein Netzwerk zu schaffen, in dem man sich gegenseitig unterstützt. Forschung im Alleingang ist schwierig. Meine Empfehlung ist, lieber kleine Studien durchzuführen und dabei auf eine gute Vorbereitung und Qualität zu achten.

Das Gespräch führte Eva Trompetter. 